\title{
A Location-to-Segmentation Strategy for Automatic Exudate Segmentation in Colour Retinal Fundus Images
}

\author{
Qing Liü,b,c, Beiji Zou, ${ }^{\mathrm{a}, \mathrm{b}}$, Jie Chen ${ }^{\mathrm{c}}$, Wei Ke ${ }^{\mathrm{c}}$, Kejuan Yue ${ }^{\mathrm{d}}$, Zailiang Chen ${ }^{\mathrm{a}, \mathrm{b}, *}$, Guoying Zhao \\ ${ }^{a}$ School of information science and engineering, Central South university, Changsha 410083, China \\ ${ }^{b}$ Ministry of Education-China Mobile Joint Laboratory For Mobile Health, Changsha 410083, China \\ ${ }^{c}$ Center for Machine Vision and Signal Analysis, University of Oulu, Oulu, 90570, Finland \\ ${ }^{d}$ Hunan First Normal University, School of Information Science and Engineering, Changsha 410205, China
}

\begin{abstract}
The automatic exudate segmentation in colour retinal fundus images is an important task in computer aided diagnosis and screening systems for diabetic retinopathy. In this paper, we present a location-to-segmentation strategy for automatic exudate segmentation in colour retinal fundus images, which includes three stages: anatomic structure removal, exudate location and exudate segmentation. In anatomic structure removal stage, matched filters based main vessels segmentation method and a saliency based optic disk segmentation method are proposed. The main vessel and optic disk are then removed to eliminate the adverse affects that they bring to the second stage. In the location stage, we learn a random forest classifier to classify patches into two classes: exudate patches and exudate-free patches, in which the histograms of completed local binary patterns are extracted to describe the texture structures of the patches. Finally, the local variance, the size prior about the exudate regions and the local contrast prior are used to segment the exudate regions out from patches which are classified as exudate patches in the location stage. We evaluate our method both at exudate-level and image-level. For exudate-level evaluation, we test our method on e-ophtha EX dataset, which provides pixel level annotation from the specialists. The experimental results show that our method achieves $76 \%$ in sensitivity and $75 \%$ in positive prediction value(PPV), which both outperform the state of the art methods significantly. For image-level evaluation, we test our method on DiaRetDB1, and achieve competitive performance compared to the state of the art methods.
\end{abstract}

Keywords: diabetic retinopathy, colour retinal fundus image, optic disk segmentation, exudate location, exudate segmentation

\section{Introduction}

Diabetic retinopathy is the main cause of blindness in working age population in the world [1]. The World Diabetes Foundation estimates that over 438 million people will suffer from diabetic retinopathy by 2030. As the window to a person's body, retinal fundus contains rich anatomical structures, such as optic disk, vessels and macula, as Fig.1(a) shows. Specifically, it provides an important manifestation of the presence of diabetic retinopathy. Traditional diagnosis process is to analyse the retinal fundus images and quantify the exudates according to the ophthalmologists' experience. It is time consuming and the quantification precision totally depends on the ophthalmologists' experience. To reduce the ophthalmologists' burden and quantify the exudates precisely, automatic exudate segmentation in the retinal fundus images becomes emergent.

Exudate on the retinal fundus is an important manifestation of the presence of diabetic retinopathy. Fig. 1 shows an example for colour retinal fundus image with exudate regions and

\footnotetext{
*I am corresponding author

Email addresses: qing. 1iu.411@gmail.com (Qing Liu), bjzou@csu.edu.cn (Beiji Zou), jiechen@ee.oulu.fi (Jie Chen), wke@ee.oulu.fi (Wei Ke), 11994602@qq.com (Kejuan Yue), zailiangchencs@gmail.com (Zailiang Chen), gyzhao@ee.oulu.fi (Guoying Zhao)
}

the pixel level annotation is provided by an ophthalmologist. In retinal fundus images, they appear as white/yellow soft structures and have variable sizes. For example, in e-ophtha EX dataset [2], the small one is only in a few pixels while the big one is up to be in thousands of pixels. Their shapes and intensities are diverse. Moreover, optic nerve fibbers and the bright reflections within the vessels exhibit similar appearance and can mislead the segmentation of exudate regions. In summary, precise and automatic exudate region segmentation faces a lot of challenges.

Benefiting from the advanced retina fundus camera, the resolution of the retinal fundus images is up to $2544 \times 1696$. However, the size of the exudate regions is extremely small compared to the whole image. Consequently, instead of detecting the exudate regions from the whole image directly, existing methods usually adopt a two-stage detection framework: candidate detection stage and refining stage. In candidate detection stage, the structures and regions which are apparently not exudate regions are removed via mathematical morphology operators. In refining stage, there are two ways to further determine whether the candidate is an exudate region or not. The first one is to learn a classifier while the other is to threshold a feature map directly. However, due to the high diversity of the size of the exudate region, it is difficult to select satisfying parameters 


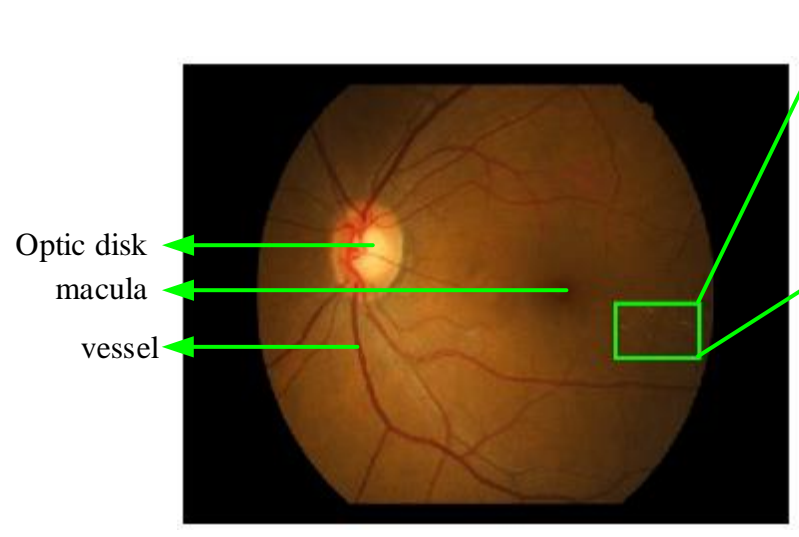

(a)

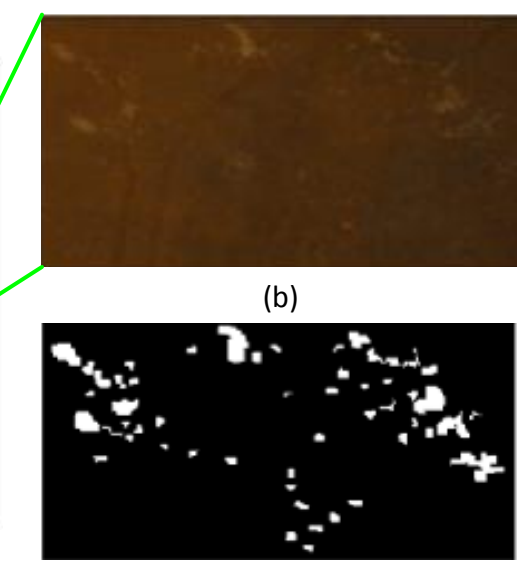

(c)

Figure 1: (a) color retinal image from the public exudates dataset e-ophtha EX, (b)zoom in to the exdudates region, (c) pixel level annotation (Best viewed in colour).

for mathematical morphology operators.

Due to the large variety of the exudates in size, intensity, shape and contrast, directly segmenting the small proportion of the exudate pixels from the whole retinal images leads to high miss detection rate. Instead of directly segmenting exudates or exudates candidate using unsupervised methods, we learn a random forest classifier to locate the exudates, then segment the exudates from the located regions. We call this strategy as location-to-segmentation strategy. In detail, it involves three stages, as seen in Fig. 2. The first stage is anatomic structures removal, in which adverse affects from the main vessels and optic disk with similar structure information to exudate regions are eliminated. Particularly, a matched filters based segmentation method is adopted to segment the main vessel. For the optic disk, we compute the saliency map to locate it and segment it based on the saliency values. Following stage is exudate location. Exudate regions exhibit brighter than their surrounding regions, so the local differences between the exudate pixels and their surrounding neighbours from the background should be positive. Additionally, the magnitudes are always large compared to the difference between two background pixels since the background regions are always smooth. Following those characteristics, we take both the sign and the magnitude of the local difference into consideration and compute the completed local binary pattern(CLBP) [3] to describe such a local texture structure. For each patch, we first compute the histograms of CLBP. Then we learn a random forest classifier to determine whether a patch is an exudate patch or exudate free patch. The third stage is exudate segmentation from the exudate patches. To further remove the non-exudate pixels in patches, local variation for each pixel in patches is computed. Size prior and regional contrast prior about the exudate regions are further used to obtain the exudate regions. We test our method on the e-ophtha EX dataset, and the experimental results show that our method outperforms the state-of-the-art method.

The contribution of this paper is three-folds: firstly, we present a location-to-segmentation strategy for exudate segmentation, which includes three stage: anatomic structures removal stage, exudate location stage and exudate segmentation stage. Secondly, we propose to use the histogram of CLBP to describe the local texture structures of the exudate regions, in which both the local difference sign and magnitude between the exudate pixel and neighbouring background pixels are considered. Thirdly, we propose an exudate region segmentation method from the located patches. Because the patches include both exudate regions and background regions and there exists high contrast between them, we exploit the size prior and regional contrast prior about the exudate regions for segmentation.

This paper is organised as follows. Section 2 reviews the existing exudates detection methods. Section 3 then illustrates the proposed method in detail. Section 4 presents the experimental results as well as the details of the experiments, including the brief introduction of public dataset for exudate detection and the quantitative evaluation. Section 5 gives the conclusion and the future work for this paper.

\section{Related Work}

Retinal fundus image analysis currently attracts lots of attention from both computer science field and ophthalmology. Its goal is to develop computational tools which will assist quantification and visualisation of the anatomical structures and lesions. It includes vessels analysis, optic disk analysis, macular analysis, micro-aneurysms detection and exudate detection. In this section, we review the existing works on exudate detection since our work mainly focus on it.

Existing detection methods can be classified to two classes: mathematical morphology based exudate detection methods and learning based exudate detection methods. Both of them include two stages: candidate detection and exudate detection from candidate.

As for mathematical morphology based exudate detection methods, vessels and optic disc are removed first, then mathematical morphology operators are performed to obtain the exudates. Akara Sopharak et al. [4] used a closing operator and reconstruction operators together with thresholding to remove the optic disk and main vessels, then discriminate the exudate 
pixels according to the local variation since the exudate pixels have high contrast to its surrounding pixels, their contour is highlighted in the standard local deviation image. Similarly, Walter et al. [5] used the grey level variation to find the exudate and determine their contours by means of morphological reconstruction techniques. In [6], the lightness $L$ of the perceptually uniform $L u v$ colour space is enhanced via a top-hat by opening operator followed by the top-hat by closing operator. Since exudates are brighter than background region, a reconstruction by dilation is performed on the regional minimal image to estimate the background region. Then subtracting the background regions from the enhanced image and performing the H-maximal transformation, the exudate candidates are obtained by a thresholding operator. Finally, a reconstruction operator is performed on the candidate regions to improve the detection accuracy. However, they ignored the local structure information of the exudate regions. The reflection from various components of the retinal tissue are erroneously regarded as exudate regions.

In learning based exudate detection methods, candidate regions are first extracted, then a feature vector for each region is extracted to learn a classifier to further determine whether the candidate is an exudate or not. In [7], a six feature combination including the pixel intensity, the standard deviation, the pixel hue, the number of edge pixels in a window, the ratio between the size of the pixels intensity cluster and the optic disc, the response to a derivative of Gaussian filter are extracted and a naive Bayes classifier is learned to detect the exudates. In [8], candidate bright-lesion areas are segmented by an improved fuzzy C-means, then a hierarchical support vector machine is learned to classify the candidate areas. In [9], a multi-scale morphological algorithm is used to obtain the candidates. Then the size, brightness, area, shape, colour and contextual information, the latter being the distance from the nearest macular are extracted to learn a support vector machine. In [10], instantaneous amplitude components are extracted from multiple frequency scales, then the exudate candidates are obtained by optically thresholding the instantaneous amplitude components. For each candidate region, colour, shape and texture features are extracted to learn a support vector machine for classification. Sánchez et al. [11] proposed a mixture models based method to dynamically threshold the images in order to separate exudates from background. In [2], not only the dark structures such as vessels and dark lesions are removed in the pre-processing stage, but also the bright structures such as the reflections and nerve fibbers are removed. Intensity feature, geometric features, textural features, hybrid feature and contextual features are extracted to learn a random forest classifier such that bright structures which are not exudates are removed. However, on one hand, there are several parameters to be determined in the first stage since several mathematical morphology operators are adopted to obtain the candidates. On the other hand, though texture features are considered in [2] [11], they are characterised by the local variance in the refining stage. In this paper, the texture features are characterised by the completed local binary pattern and used to locate the exudate regions, which is totally different from the existing works.

\section{The Proposed Method}

The proposed exudate segmentation method involves three stages: 1) anatomic structures removal, in which the field of view is extracted while the main vessels and the optic disk in the field of view are removed; 2) exudate region location in which the patches containing exudate regions are identified; 3) exudate region segmentation from the patches. Fig. 2 illustrates the framework of the proposed method.

\subsection{Anatomic structures removal}

This stage consists of three main steps: field of view segmentation, main vessel segmentation and optic disk segmentation. Fig. 3 shows the results of each step.

\section{Field of view segmentation}

Since there is no information out of the field of view, we first segment the field of view. The region out of the field of view is almost black while the field of view looks bright. There exists obvious high contrast between them. Hence, given an retinal fundus image, we first compute the gradient image. We then obtain the contour of the field of view via thresholding the gradient image and obtain the field of view. Fig. 3(b) shows an example of the field of view.

\section{Main vessels segmentation}

As the only part of blood circulation system that can be observed directly and the clinical diagnose basis for diabetes, arteriosclerosis etc, vessel detection in retinal fundus images gains lots of researchers' attention. In [12], many vessels detection methods are reviewed. The contrasts between the small vessels and their surrounding regions are too low thus mislead the exudates location. Therefore, we mainly focus on the segmentation of the main vessels.

The cross section of the retinal vessels is like the profile of a Gaussian function [13] and the intensity on the vessel's centre line is lowest. Meanwhile, they have highest contrast in green channel. Naturally, we use a Gaussian filter to match the Gaussian-like vessels in the inverted green channel of the retinal fundus image and obtain a response image, in which the responses on the vessels are stronger than the background regions. Since the original green image has contrast, we add it to the response image. Then we threshold the response image using the Otus' methods [14]. Finally, we use the close operator to remove the noise in the thresholded image and obtain the main vessels in the retinal fundus image. Fig. 3(c) shows an example of the field of view.

\section{Optic disk segmentation}

The optic disk location and segmentation is very important in many computer aided diagnosis. Though classification based optic detection methods are proposed in [15][16][17], they are data dependent. In this section, we propose an unsupervised optic disk location and segmentation method. The optic disk is a bright region in the retinal fundus image and it has high contrast to the rest image part. Hence, it attracts our humans attention most than other regions. From the view of saliency 

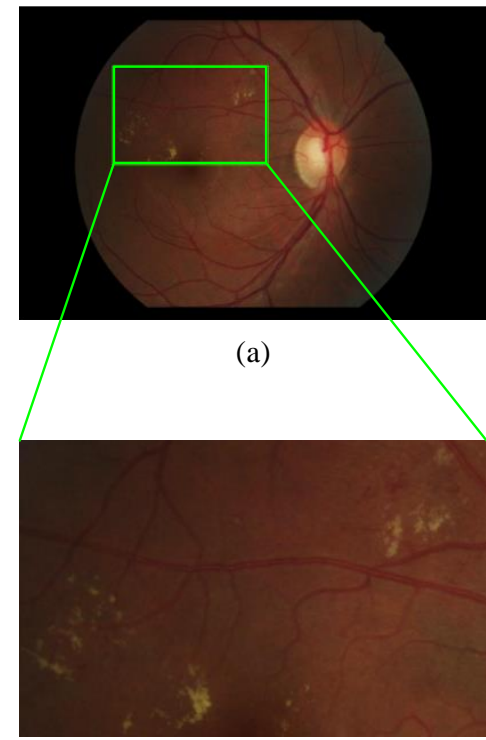

(e)

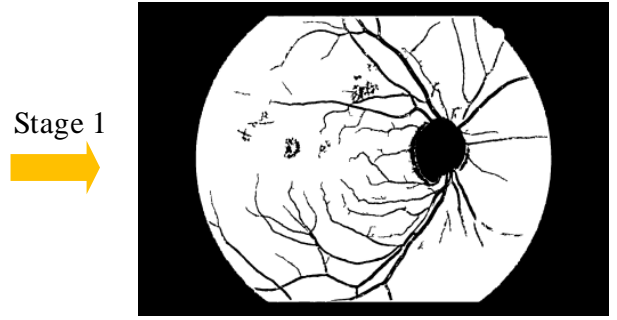

(b)

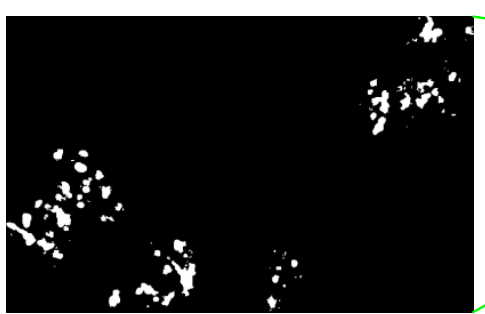

(f)

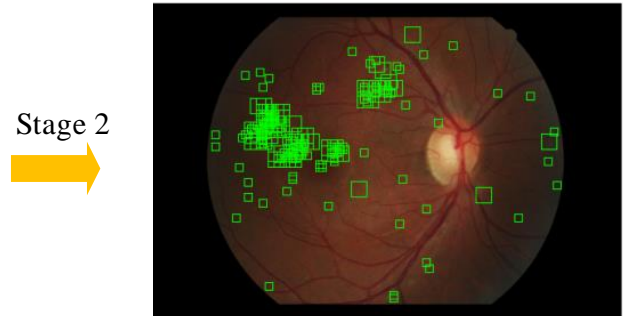

(c) Stage 3

Figure 2: The framework of the proposed method (Best viewed in colour). (a) the input colour retinal fundus image,where exudate regions are in green box. (b) the field of view part in which the optic disc and the vessels are removed. (c) the detected exudate patches obtained by the proposed exudate location method. (d) the final segmentation result, in which the white regions are the exudate regions. (e) zooming into the exudate regions in the original image. (f) zooming into the regions detected as exudate regions.

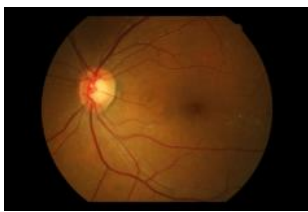

(a)

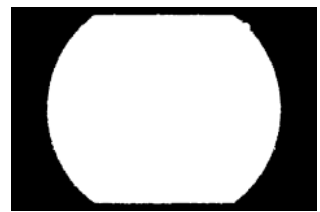

(b)

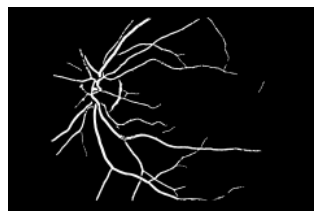

(c)

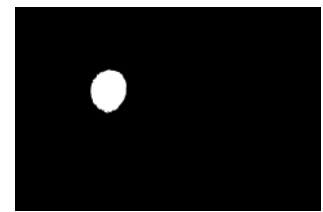

(d)

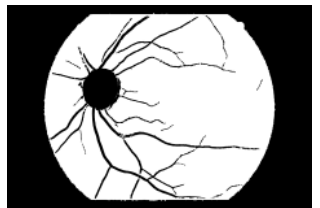

(e)

Figure 3: (a) the input image. (b) the field of view detected from (a). (c) the main vessels detected from (a). (d) the optic disk detected from (a). (e) the field of view in which the main vessels and the optic disk are removed (Best viewed in colour).

detection, it is more salient than other regions. Therefore, we use the most salient pixels to location the optic disk and use the saliency values to segment the optic disk.

First we generate a saliency map $S$ for the retinal fundus image $I$, as shown in Fig. 4(b). There are numerous methods for saliency detection from natural scene images, for example [18] [19] [20]. However, unlike the natural scene images, centre-bias prior which is used in most of the saliency detection methods is not valid in retinal fundus images. Meanwhile, the contents in the retinal fundus images are fixed compared to the complex natural scenes. Hence, simple global contrast based saliency detection methods are enough to highlight the optic disk uniformly with clear boundary. Therefore, we adopt the frequency-tuned salient region detection method (FT) [21] to generate the saliency maps for retinal fundus images.

Given an image I, we compute the saliency value $S(x, y)$ for each pixel in the field of view by:

$$
S(x, y)=\left\|\mathbf{I}_{\mu}-\mathbf{I}_{w h c}(x, y)\right\|
$$

where $\mathbf{I}_{\mu}$ is the mean colour feature vector of pixels in the filed of view. Same to FT [21], we use the $L a b$ colour space.
$\mathbf{I}_{w h c}(x, y)$ is the corresponding image pixel vector value in the Gaussian blurred version of the original image, $\|\cdot\|$ is the $L_{2}$ norm.

To reduce the adverse affects from the bright regions such as the exudate regions, instead of directly thresholding the saliency map $S$, we locate the optic disk in the saliency map first according to the most salient regions in $S$. Thereafter, we segment the optic disk $T_{O D}$ from a small window by thresholding. Finally, we fill in the holes in $T_{O D}$ and decide whether the segment we obtain $T_{O D}$ is the optic disk or not according to the area of the segmented region. If the area of the segment is too large or too small, we classify it as a background region. Otherwise, we consider it as the optic disk. Fig. 4 illustrates the results of each step.

\subsection{Exudate location}

In this section, we propose a novel learning based exduate location method. The exudates appear as bright structures and have high contrast compared to the local background regions. Obviously, the intensity of a pixel from an exudate region is larger than its local neighbours from the background, and it ex- 


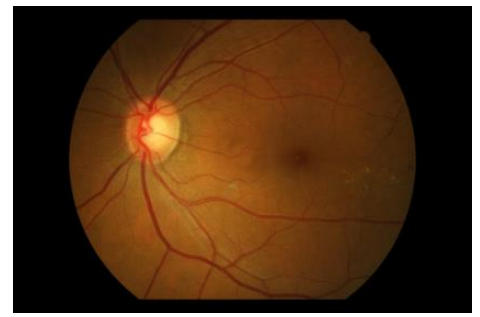

(a)

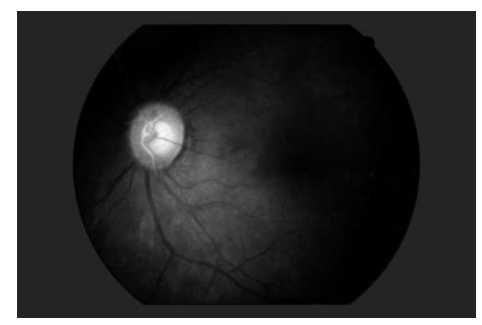

(b)

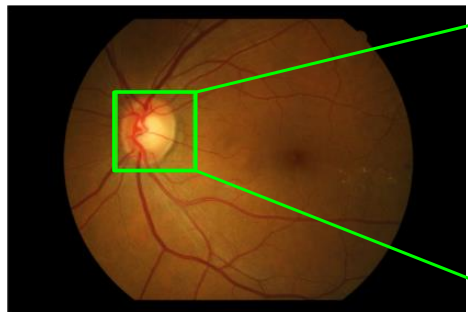

(c)

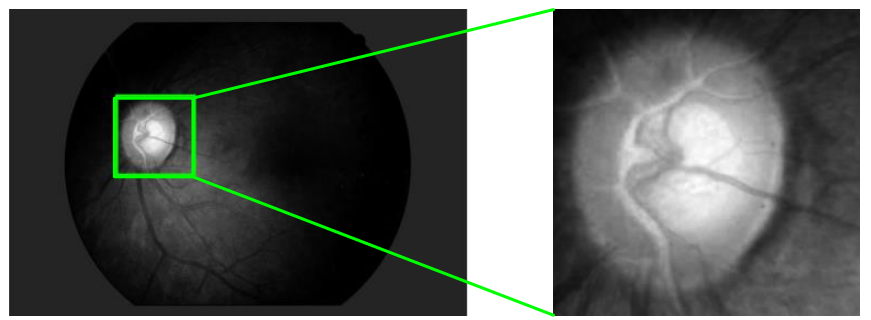

(d)

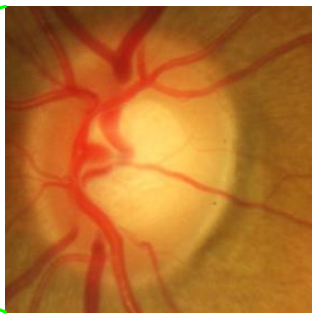

(e)

(f)

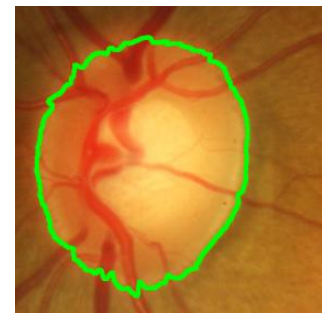

(g)

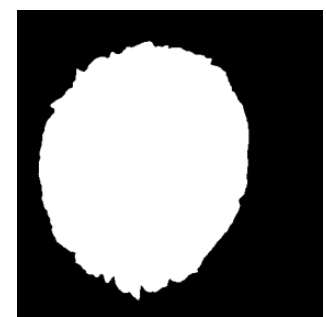

(h)

Figure 4: Illustration for optic disk segmentation (Best viewed in colour). (a) input image. (b) saliency map. (c) optic disk location on the original image. (d) optic disk location on the saliency map. (e-f) zooming into the optic disc region. (g) the detected contour of the optic disk. (h) segmentation of the optic disk via thresholding (f).

hibits different texture structures to pixels from the background region. To describe the characteristics of the exudate regions, we first compute the histograms of CLBP for each patch $P$ in the image.

Given a pixel $g_{c}$ in the patch $P$ and its $N$ circularly and evenly spaced neighbours $g_{n}, n \in[0, \cdots, N-1]$, the original local binary pattern (LBP) [22] encodes the sign $s_{n}$ of its local difference to neighbours $d_{n}=g_{n}-g_{c}$ by:

$$
L B P_{N, R}=\sum_{n=0}^{N-1} s_{n} \cdot 2^{n}
$$

where $s_{n}=\operatorname{sign}\left(d_{n}\right)$ and $\operatorname{sign}(\cdot)$ is a sign function. $s_{n}=1$ if $d_{n} \geq 0$, otherwise $s_{n}=0 . R$ is the radius of the neighbourhood.

In CLBP, the grey level $g_{c}$, the sign of its local difference as well as the magnitude are considered. Its local difference $d_{p}$ is decomposed into two complementary components via a signmagnitude transform(LDSMT):

$$
d_{n}=s_{n} * m_{n} \text { and }\left\{\begin{array}{l}
s_{n}=\operatorname{sign}\left(d_{n}\right) \\
m_{n}=\left|d_{n}\right|
\end{array}\right.
$$

where $s_{n}$ is the sign component, $m_{n}$ is the magnitude component, $\operatorname{sign}(\cdot)$ is a sign function. For the sign component, the original LBP operator i.e. Eq. 2 is used to encode it, denoted by CLBP_S. For the magnitude component, the same encoding strategy is adopted after binarizing the magnitude component:

$$
C L B P \_M=\sum_{n=0}^{N-1} \operatorname{sign}\left(m_{n}-c\right) \cdot 2^{n}
$$

where $\operatorname{sign}\left(m_{n}-c\right)=1$ if $m_{n}-c \geq 0$, otherwise $\operatorname{sign}\left(m_{n}-c\right)=0$. $c$ is a threshold. We set it as the mean local difference $m_{n}$ of the patch in our experiments. Similarly, CLBP_S and CLBP_M are mapped to the uniform patterns with rotation invariant[22] via a lookup table. Meanwhile, CLBP also takes the discriminant ability of the centre pixel into consideration, and encodes the sign of its difference to the mean intensity of the patch by CLBP_Centre (CLBP_C) operator.

As to the combination, the CLBP_M and CLBP_C are combined by a $2 \mathrm{D}$ joint histogram first, and then converted to a 1D histogram CLBP_M/C. Finally, CLBP_S and CLBP_M/C are concatenated into a joint histogram CLBP_S_M/C. Since the exudates appear as white or yellow soft structures, we take both the red and green colour channels into consideration, and obtain the feature vector $\left[C L B P_{-} S_{-} M / C_{r}, C L B P_{-} S_{-} M / C_{g}\right]$ for the patch $P$.

Given the feature vectors of the exudate patches and the feature vectors of the exudate-free patches, we learn a random forest classifier to determine whether a patch is an exudate patch or an exudate-free patch. The number of trees is set to five and the maximum depth is set to sixteen. Up to now, we obtain the candidate exudate patches. Fig. 5 shows two examples. For a better visualisation, the exudate regions are labelled as red, and the patches really contains exudate regions are labelled by green windows while the exudate free patches which are erroneously detected as exudate patches are labelled by the yellow windows.

\subsection{Exudate segmentation}

To further segment the exudate regions from the exudate candidate patches and remove the exudate-free patches which are erroneously detected as the exudate patches, we first compute the local variation $V$ for each pixel $x \in p_{i}$ to highlight the closely distributed cluster of exudate pixels:

$$
V(x)=\frac{1}{\left|p_{i}\right|-1} \cdot \sum_{j \in p_{i}}\left(p_{i}(j)-\mu_{p_{i}}(x)\right)^{2}
$$



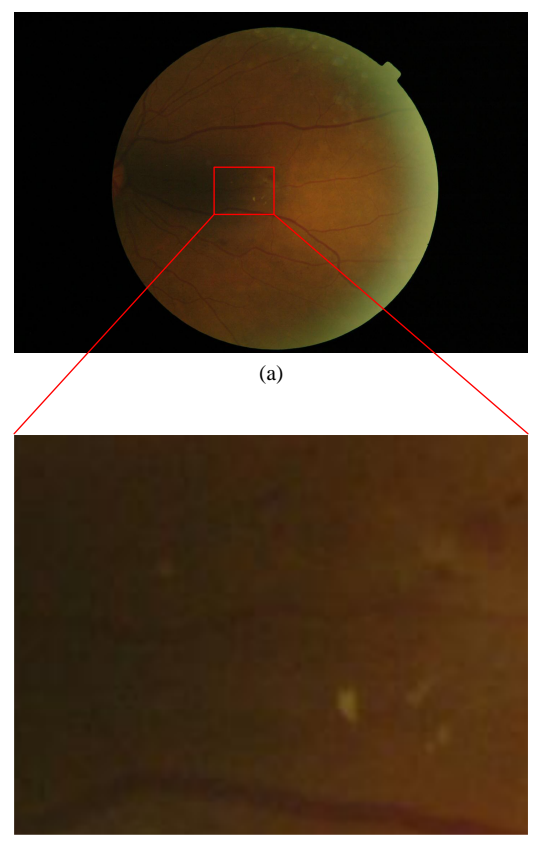

(c)
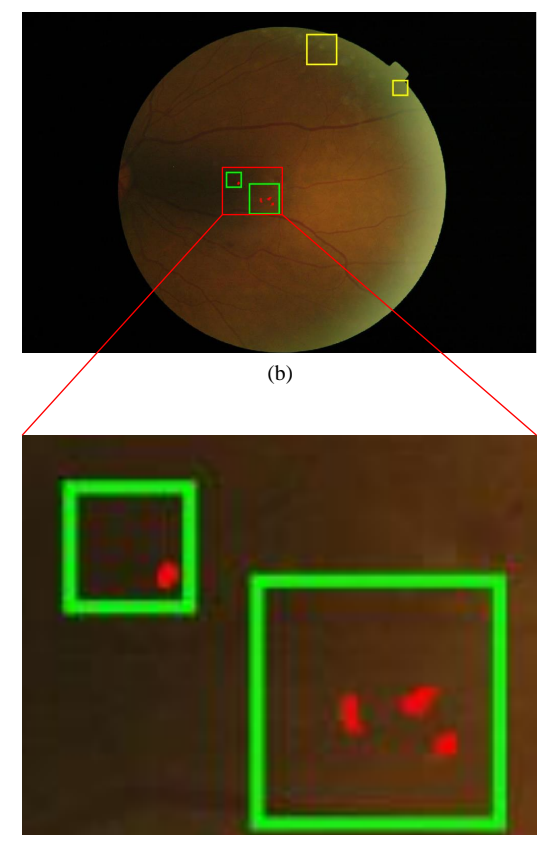

(d)

Figure 5: An example for exudate location (Best viewed in colour). (a) is the input image. (b) is the visualisation of the location result, in which the exudates are labelled in red, the exudate patches are labelled by green windows, and the exudate free patches which are erroneously detected as exudates are labelled by the yellow windows. (c) is the detail view of the red window in (a). (d) is the detail view of the red window in (b).

where $\left|p_{i}\right|$ is the pixel number of patch $p_{i}, p_{i}(j)$ is the $j$-th pixel's intensity of the green colour channel and $\mu_{p_{i}}(x)$ is the mean intensity of a window centred on $x$. Then we threshold the local variation $V$ to obtain the exudates pixels mask $V_{T}$ :

$$
V_{T}=V \odot f(V ; \alpha)
$$

in which $\odot$ is the dot product operator. $f(V(x) ; \alpha)$ is an indicator function. If $V(x)>\alpha$, then $f(V(x) ; \alpha)=1$, otherwise $f(V ; \alpha)=0 . \alpha$ is a adaptively determined by Otsu's method. The exudate pixels have brighter intensity than the background pixels. Hence, pixels with bright intensity should be exudate pixels:

$$
E(x)= \begin{cases}1 & \text { if } V_{T}(x)=1 \text { or } I(x)>\mu_{V_{T}} \\ 0 & \text { otherwise }\end{cases}
$$

where $I(\cdot)$ is intensity of the green colour channel, $\mu_{V_{T}}$ is the mean intensity in green colour channel of the non-zero pixels in $V_{T}$.

To further determine whether the pixels in $E$ are the exudate pixels, two priors about the exudates are used. One is the size prior, and the other is the local contrast prior. For the size prior, we assume that the region should belong to background if it is too large. So such patches should be removed:

$$
E_{s}= \begin{cases}E & \text { if } \sum_{j \in E} E(j)<T_{\text {size }} \cdot|E| \\ 0 & \text { otherwise }\end{cases}
$$

where $T_{\text {size }}$ is a ratio threshold. For the local contrast prior, we assume that the exudate regions have higher intensity than the background regions:

$$
E_{c}= \begin{cases}E & \text { if } \mu_{E}-\mu_{\bar{E}}>T_{\text {contrast }} \\ 0 & \text { otherwise }\end{cases}
$$

where $\mu_{E}$ is the mean intensity in green colour channel of the non-zero pixels in $E_{4}$, and $\mu_{\bar{E}}$ is the mean intensity in green colour channel of the zero pixels in $E, T_{\text {contrast }}$ is a threshold for the local contrast to the background pixels. Up to now, the final exudate regions are obtained by $E_{\text {final }}=E_{s} \odot E_{c}$.

\section{Experimental results}

In this section, we evaluate our method at two levels: exudate-level and image-level. Exudate-level evaluation measures the capability of segmentation methods to segment the exudates from retinal images precisely. Image-level evaluation measures the capability of methods to discriminate whether a retinal image contains exudates. Accordingly, e-ophtha EX dataset with precisely labelled ground truth is used for exudatelevel evaluation and DiaRetDB1 with roughly labelled ground truth is used for image-level evaluation. E-ophtha EX dataset is the only public available database to provide pixel level annotation for exudate segmentation. It contains 47 images with exudate regions and 35 exudate-free images. Since we focus on exudate segmentation, we only use 47 images in our experiments. DiaRetDB1 [23] contains 89 images. Its test part includes 61 images in which 29 images contain exudates. Each image is roughly labelled by four specialists. Regions labelled by at least three specialists are regarded as exudate regions. Fig. 7 shows an example of retinal image from DiaRetDB1.

\subsection{Evaluation on e-ophtha EX dataset}

We use the number of True Positive pixels (TP), the number of False Positive pixels (FP), the number of False Negative pixels (FN), the Sensitivity (S) and the Positive Prediction 


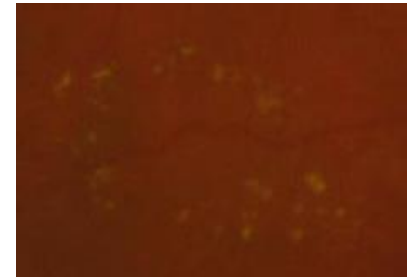

(a1)

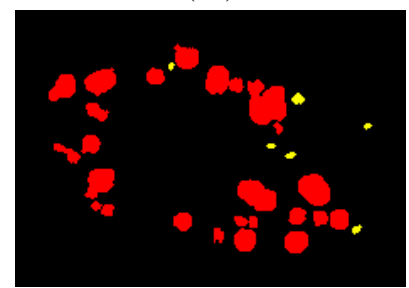

(a2)

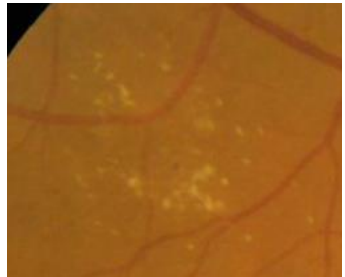

(b1)

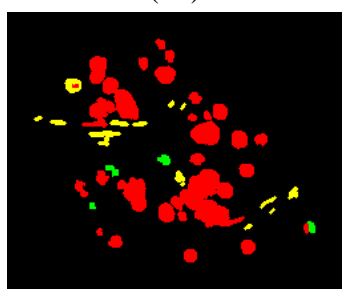

(b2)

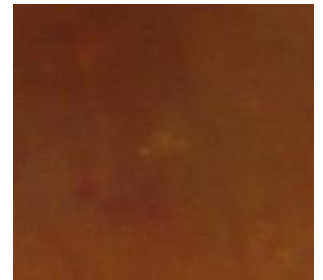

(c1)

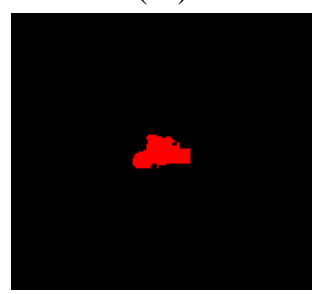

(c2)

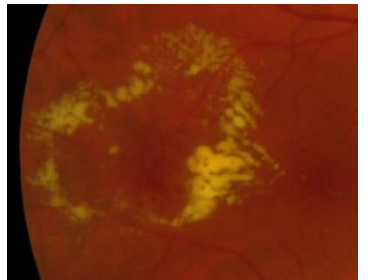

(d1)

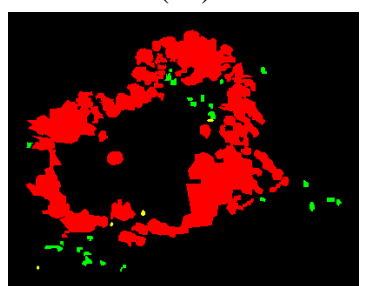

(d2)

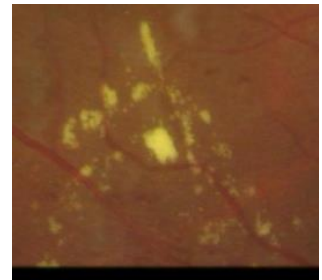

(e1)

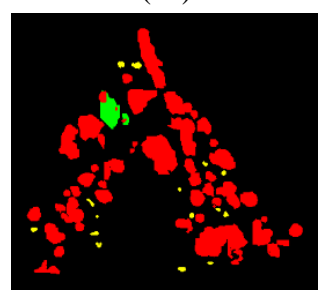

(e2)

Figure 6: Example of pixel level validation (Best viewed in colour). (a1-e1) Original exudates region cropped from the retinal fundus images. (a2-e2) Results of pixel level validation with $\sigma=0.2, T_{\text {size }}=0.4, T_{\text {contrast }}=6$. The red pixels are the TP pixels, the green pixels are the FN pixels, the yellow pixels are the FP pixels and the black pixels are the TN pixels.

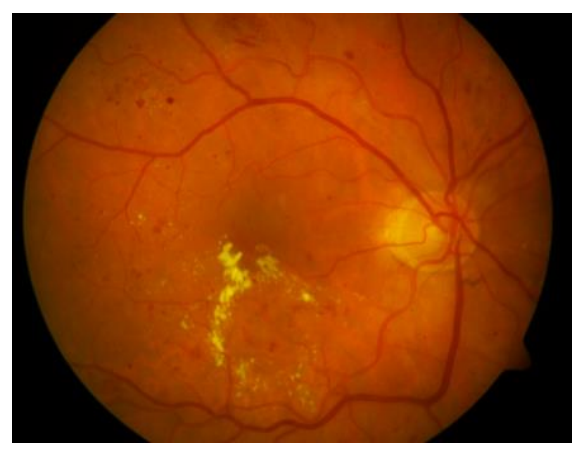

(a)

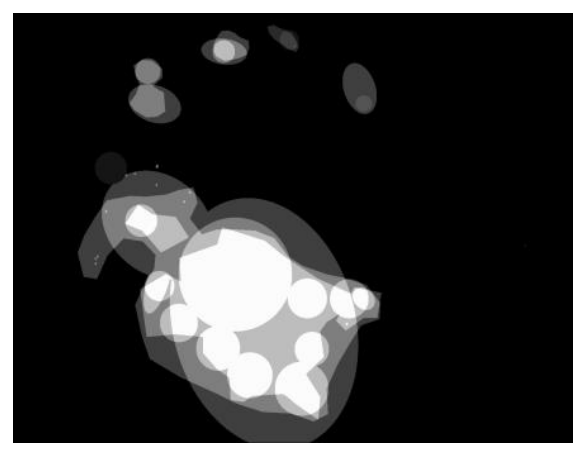

(b)

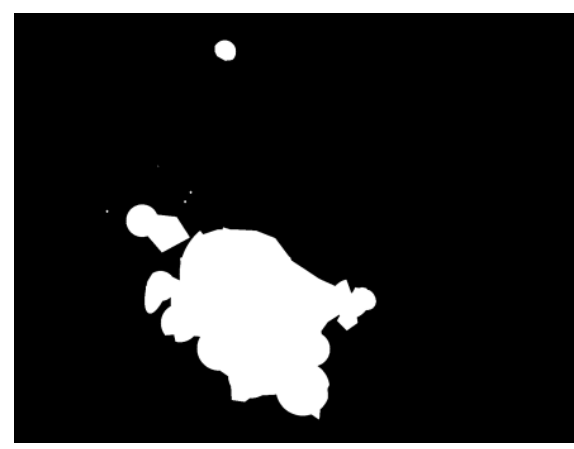

(c)

Figure 7: Example of retinal images in DiaRetDB1 (Best viewed in colour). (a) Original retinal image. (b) Exudate regions labelled by four eye specialists. (c) Exudate regions labelled by at least three eye specialists.

Value (PPV) to quantitatively evaluate the proposed method.

Given the segmented exudate connected component set $\left\{D_{1}, D_{2}, \cdots, D_{N}\right\}$ and the ground truth exudate component set $\left\{G_{1}, G_{2}, \cdots, G_{M}\right\}$, following [2], if a pixel belongs to

$$
\{D \cap G\} \cup\left\{D_{i} \mid \frac{\left|D_{i} \cap G\right|}{\left|D_{i}\right|}>\sigma\right\} \cup\left\{G_{j} \mid \frac{\left|G_{j} \cap D\right|}{\left|G_{j}\right|}>\sigma\right\}
$$

it is considered as a TP pixels, where $D=D_{1} \cup D_{2} \cup \cdots \cup D_{N}$ is the mask of the detected exudates, $G=G_{1} \cup G_{2} \cup \cdots \cup G_{M}$ is the mask of the ground truth exudates, and $|\cdot|$ is the cardinal of a set. $\sigma$ is a parameter ranging from 0 to 1 . In [24], it is set to 0 , which means that a connected region is considered as TP if it overlaps at least in part with the ground-truth. However, a large segmentation mask touching an exudate will get excellent results, which is actually inappropriate. Hence, following [2], we set $\sigma$ to 0.2 . If a pixel belongs to

$$
\left\{D_{i} \mid D_{i} \cap G=\emptyset\right\} \cup\left\{D_{i} \cap \bar{G} \mid \frac{\left|D_{i} \cap G\right|}{\left|D_{i}\right|} \leq \sigma\right\}
$$

it is considered as a FP pixel. And it will be considered as a FN pixel if it belongs to the following set

$$
\left\{G_{j} \mid G_{j} \cap D=\emptyset\right\} \cup\left\{G_{j} \cap \bar{D} \mid \frac{\left|G_{j} \cap G\right|}{\left|G_{j}\right|} \leq \sigma\right\}
$$

The rest pixels are considered as TN pixels.

Given the TP, FN and FP, the sensitivity $S$, indicating the ratio of exudate pixels correctly marked as exudate pixels, is defined by:

$$
S=\frac{T P}{T P+F N}
$$

The PPV is defined by:

$$
P P V=\frac{T P}{T P+F P}
$$

which indicates the ratio of detected exudate pixels annotated as exudate pixels by specialists. Considering both the sensitivity and PPV, their harmonic mean $F-$ score is computed by:

$$
F-\text { score }=\frac{2 \times S \times P P V}{S+P P V}
$$




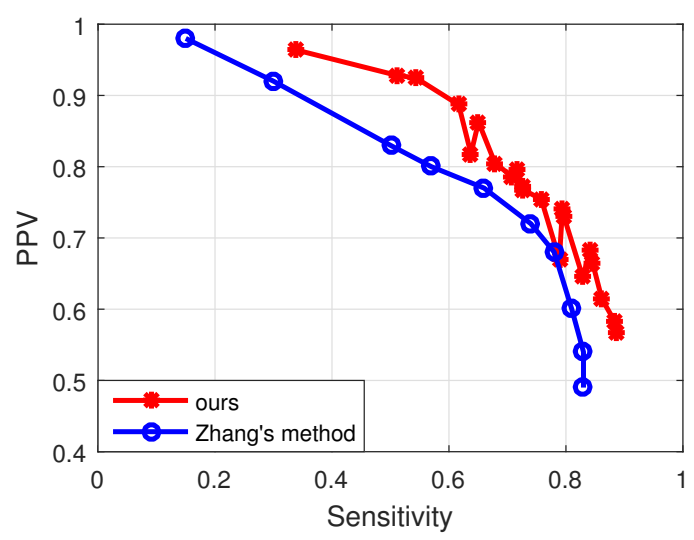

Figure 8: PPV-sensitivity curves of the proposed method and Zhang's method [2].

In the location stage, to obtain a segmentation result for each image in the dataset, we divide it into three subsets with equal size. When we test on one subset, we train the random forest classifier on the other two subsets. The patch size is set to 41 . The positive samples consist of patches which are centred on the exudates' centres while the negative samples are randomly sampled from the background regions which do not contain any exudate pixel.

In the segmentation stage, there are two parameters : $T_{\text {size }}$ and $T_{\text {contrast }}$. We vary $T_{\text {size }}\left(T_{\text {size }}=0.2,0.3,0.4,0.45\right)$ and $T_{\text {contrast }}\left(T_{\text {contrast }}=0,3,6,9,12\right)$. The results are reported in Table.1. As we can see from Table. 1 , when we increase $T_{\text {size }}$, the sensitivity increases while the PPV decreases. On the contrast, when we increase $T_{\text {contrast }}$, the sensitivity decreases while the PPV increases. Taking both the sensitivity and PPV into consideration, we set $T_{\text {size }}=0.4$ and $T_{\text {contrast }}=6$. Under this setting, our method achieves $76 \%$ in sensitivity and $75 \%$ in PPV. Fig. 6 shows some segmentation results. To visualise the results more clearly, we only show the exudates parts cropped from the retinal fundus images rather than the whole images. Fig. 6 (a1-e1) are the exudates parts cropped from five retinal fundus images. Fig. 6 (a2-e2) are the segmentation results, in which the red pixels are the TP pixels, the green pixels are the FN pixels, the yellow pixels are the FP pixels and the black pixels are the TN pixels. As we can see that some small exudates are missed by the proposed method because of their low contrasts, but most of the large exudates can be segmented well. Furthermore, the proposed method perform well when the retinal fundus contains only a single exudate as Fig. 6 (c1-c2) shows.

First, we compare the PPV-Sensitivity curves between ours and Zhang's method [2] in Fig. 8. Zhang's method [2] obtains a probability map from a support vector machine, and different segmentation can be generated when thresholding the probability map by different thresholds. The data for the curve of Zhang's method [2] are from their paper. As it shows, the proposed method can achieve better PPV under the same sensitivity. Additionally, as a comparative study, we implement [5] and [6] and try our best to achieve their best performances. In Table.
2, we report the performances of [2], [5], [6] and our method. As it shows, the proposed method outperforms the state-of-thearts significantly.

\subsection{Evaluation on DiaRetDB1}

To demonstrate the capability of our method to discriminate whether a retinal image contains exudates, we validate our method on DiaRetDB1 at image level. As we can see from Fig. 7 , the ground truth in DiaRetDB1 is too rough to train our exudate location model on DiaRetDB1. Thus we use the trained model on e-ophtha EX to test the images from DiaRetDB1. We use sensitivity, specificity and accuracy as image-level evaluation metrics. We consider the segmentation result for an image as a TP if the input image contains exudates according to both our segmentation method and the ground truth. The segmentation result for an image is considered as a TN if the input image does not contain exudates according to both our segmentation method and the ground truth. The segmentation result is considered as FP when the image does not contain exudate according to the ground truth while our segmentation method segments exudates from the image. We had a FN case in the reverse case. Based on such definitions, we use Eq. (10) to compute the sensitivity to indicate the true positive rate and Eq. (11) to compute the specificity to indicate the true negative rate. As for the accuracy, we compute it by

$$
\text { Accuaracy }=\frac{T P+T N}{T P+T N+F P+F N}
$$

Table 3. shows the comparative results between the proposed method and other methods on DiaRetDB1 at image level. Though [5] and [6] performs better in sensitivity than ours, our method performs better when the images do not contain exudates. Overall, our method achieves higher accuracy than [5] and [6].

\section{Conclusions and future work}

In this paper, a location-to-segmentation strategy is presented to segment the exudates in retinal fundus images. It involves three stages. The first stage, i.e. pre-process stage, can provide the visualisation for the main anatomic structures such as the optic disk and the main vessels. In the second stage, the local structure of the exudates is described via CLBP. A random forest classifier is learned to locate the exudate candidates. In third stage, the local variance, exudate size prior and local contrast prior are used to segment the exudate regions from each patch. The proposed method detects and quantifies the exudate regions, and further facilitates for the ophthalmologists in the diabetic retinopathy screening and diagnosis process. The experimental results on the public exudate detection data set show its superiority both at exudate-level evaluation and image-level evaluation compared to the state-of-the-art method.

For the feature work, on one hand, we plan to learn an adaptive threshold for CLBP_M operator to improve its 
Table 1: The results of the proposed methods when $T_{\text {size }}$ and $T_{\text {contrast }}$ take different values.

\begin{tabular}{|c|c|c|c|c|c|c|c|c|}
\hline$T_{\text {contrast }}$ & \multicolumn{2}{|c|}{$\mathrm{T}_{\text {size }}=0.2$} & \multicolumn{2}{c|}{$\mathrm{T}_{\text {size }}=0.3$} & \multicolumn{2}{c|}{$\mathrm{T}_{\text {size }}=0.4$} & \multicolumn{2}{c|}{$\mathrm{T}_{\text {size }}=0.45$} \\
\hline & Sensitivity & PPV & Sensitivity & PPV & Sensitivity & PPV & Sensitivity & $\mathrm{PPV}$ \\
\hline 0 & $73 \%$ & $77 \%$ & $80 \%$ & $73 \%$ & $85 \%$ & $66 \%$ & $89 \%$ & $57 \%$ \\
\hline 3 & $72 \%$ & $77 \%$ & $79 \%$ & $74 \%$ & $84 \%$ & $68 \%$ & $88 \%$ & $58 \%$ \\
\hline 6 & $71 \%$ & $79 \%$ & $72 \%$ & $80 \%$ & $76 \%$ & $75 \%$ & $86 \%$ & $61 \%$ \\
\hline 9 & $68 \%$ & $81 \%$ & $62 \%$ & $89 \%$ & $65 \%$ & $86 \%$ & $83 \%$ & $65 \%$ \\
\hline 12 & $64 \%$ & $82 \%$ & $51 \%$ & $93 \%$ & $54 \%$ & $92 \%$ & $79 \%$ & $67 \%$ \\
\hline
\end{tabular}

Table 2: Comparative results for exudate-level validation for the proposed method on e-ophtha EX dataset.

\begin{tabular}{|c|c|c|c|}
\hline & Sensitivity & PPV & F-score \\
\hline Zhang et al. [2] & $74 \%$ & $72 \%$ & $73 \%$ \\
\hline Walter et al. [5] & $44 \%$ & $65 \%$ & $52 \%$ \\
\hline Welfer et al. [6] & $79 \%$ & $55 \%$ & $69 \%$ \\
\hline Proposed method & $\mathbf{7 6 \%}$ & $\mathbf{7 5 \%}$ & $\mathbf{7 6 \%}$ \\
\hline
\end{tabular}

Table 3: Comparative results for image level validation for the proposed method on the dataset DiaRetDB1.

\begin{tabular}{|c|c|c|c|}
\hline & Sensitivity & Specificity & Accuracy \\
\hline Walter et al. [5] & $86 \%$ & $69 \%$ & $77 \%$ \\
\hline Welfer et al. [6] & $100 \%$ & 0 & $48 \%$ \\
\hline Proposed method & $\mathbf{8 3 \%}$ & $\mathbf{7 5 \%}$ & $\mathbf{7 9 \%}$ \\
\hline
\end{tabular}

discriminative ability for exudate location. In this way, the missing detection rate can be further reduced. On the other hand, exudate segmentation is one task in a computer aided diagnosis system or screening system. To build such a system, we will focus on other retinal image processing tasks such as vessels segmentation and quantification for vessel's width, curvature etc.

\section{Acknowledgements}

Q. Liu and W. Ke were partially supported by the scholarship from China Scholarship Council (CSC). B. Zou and Z. Chen were partially supported by the National Natural Science Foundation of China under Grant No.61573380 and No. 61440055. G. Zhao and J. Chen were partially supported by Academy of Finland, Tekes Fidipro Program and Infotech Oulu. K. Yue was partially supported by Scientific Research Fund of Hunan Provincial Education Department under Grant No.13C143.

\section{References}

[1] K. Lim, Registration of new blindness in singapore for 1985-1995., Singapore medical journal 39 (3) (1998) 104-106.

[2] X. Zhang, G. Thibault, E. Decenciere, B. Marcotegui, B. Laÿ, R. Danno, G. Cazuguel, G. Quellec, M. Lamard, P. Massin, et al., Exudate detection in color retinal images for mass screening of diabetic retinopathy, Medical image analysis 18 (7) (2014) 1026-1043.
[3] Z. Guo, L. Zhang, D. Zhang, A completed modeling of local binary pattern operator for texture classification, Image Processing, IEEE Transactions on 19 (6) (2010) 1657-1663.

[4] A. Sopharak, B. Uyyanonvara, S. Barman, T. H. Williamson, Automatic detection of diabetic retinopathy exudates from non-dilated retinal images using mathematical morphology methods, Computerized Medical Imaging and Graphics 32 (8) (2008) 720-727.

[5] T. Walter, J.-C. Klein, P. Massin, A. Erginay, A contribution of image processing to the diagnosis of diabetic retinopathy-detection of exudates in color fundus images of the human retina, Medical Imaging, IEEE Transactions on 21 (10) (2002) 1236-1243.

[6] D. Welfer, J. Scharcanski, D. R. Marinho, A coarse-to-fine strategy for automatically detecting exudates in color eye fundus images, Computerized Medical Imaging and Graphics 34 (3) (2010) 228-235.

[7] A. Sopharak, K. T. New, Y. A. Moe, M. N. Dailey, B. Uyyanonvara, Automatic exudate detection with a naive bayes classifier, in: International Conference on Embedded Systems and Intelligent Technology, Bangkok, Thailand, 2008, pp. 139-142.

[8] Z. Xiaohui, O. Chutatape, Detection and classification of bright lesions in color fundus images, in: Image Processing, 2004. ICIP’04. 2004 International Conference on, Vol. 1, IEEE, 2004, pp. 139-142.

[9] A. D. Fleming, S. Philip, K. A. Goatman, G. J. Williams, J. A. Olson, P. F. Sharp, Automated detection of exudates for diabetic retinopathy screening, Physics in medicine and biology 52 (24) (2007) 7385.

[10] C. Agurto, V. Murray, H. Yu, J. Wigdahl, M. Pattichis, S. Nemeth, E. S. Barriga, P. Soliz, A multiscale optimization approach to detect exudates in the macula, Biomedical and Health Informatics, IEEE Journal of 18 (4) (2014) 1328-1336.

[11] C. I. Sánchez, M. García, A. Mayo, M. I. López, R. Hornero, Retinal image analysis based on mixture models to detect hard exudates, Medical Image Analysis 13 (4) (2009) 650-658.

[12] M. M. Fraz, P. Remagnino, A. Hoppe, B. Uyyanonvara, A. R. Rudnicka, C. G. Owen, S. A. Barman, Blood vessel segmentation methodologies in retinal images-a survey, Computer methods and programs in biomedicine 108 (1) (2012) 407-433.

[13] F. Zana, J.-C. Klein, Segmentation of vessel-like patterns using mathematical morphology and curvature evaluation, Image Processing, IEEE Transactions on 10 (7) (2001) 1010-1019.

[14] N. Otsu, A threshold selection method from gray-level histograms, Automatica 11 (285-296) (1975) 23-27.

[15] J. Cheng, J. Liu, Y. Xu, F. Yin, D. W. K. Wong, N.-M. Tan, D. Tao, C.-Y. Cheng, T. Aung, T. Y. Wong, Superpixel classification based optic disc and optic cup segmentation for glaucoma screening, Medical Imaging, IEEE Transactions on 32 (6) (2013) 1019-1032.

[16] F. Yin, J. Liu, S. H. Ong, Y. Sun, D. W. Wong, N. M. Tan, C. Cheung, M. Baskaran, T. Aung, T. Y. Wong, Model-based optic nerve head segmentation on retinal fundus images, in: Engineering in Medicine and Biology Society, EMBC, 2011 Annual International Conference of the IEEE, IEEE, 2011, pp. 2626-2629.

[17] D. Wong, J. Liu, N. Tan, F. Yin, B. Lee, T. Wong, Learning-based approach for the automatic detection of the optic disc in digital retinal fundus photographs, in: Engineering in Medicine and Biology Society (EMBC), 2010 Annual International Conference of the IEEE, IEEE, 2010, pp. 5355-5358.

[18] Y. Wei, F. Wen, W. Zhu, J. Sun, Geodesic saliency using background priors, in: Computer Vision-ECCV 2012, Springer, 2012, pp. 29-42. 
[19] W. Zhu, S. Liang, Y. Wei, J. Sun, Saliency optimization from robust background detection, in: Computer Vision and Pattern Recognition (CVPR), 2014 IEEE Conference on, IEEE, 2014, pp. 2814-2821.

[20] B. Zou, Q. Liu, Z. Chen, S. Liu, X. Zhang, Saliency detection using boundary information, Multimedia Systems (2015) 1-9.

[21] R. Achanta, S. Hemami, F. Estrada, S. Susstrunk, Frequency-tuned salient region detection, in: Computer vision and pattern recognition, 2009. cvpr 2009. ieee conference on, IEEE, 2009, pp. 1597-1604.

[22] T. Ojala, M. Pietikäinen, T. Mäenpää, Multiresolution gray-scale and rotation invariant texture classification with local binary patterns, Pattern Analysis and Machine Intelligence, IEEE Transactions on 24 (7) (2002) 971-987.

[23] R. V. J. P. H. Kälviäinen, H. Uusitalo, Diaretdb1 diabetic retinopathy database and evaluation protocol, Medical Image Understanding and Analysis 2007 (2007) 61.

[24] L. Giancardo, F. Meriaudeau, T. P. Karnowski, Y. Li, K. W. Tobin Jr, E. Chaum, Automatic retina exudates segmentation without a manually labelled training set, in: Biomedical Imaging: From Nano to Macro, 2011 IEEE International Symposium on, IEEE, 2011, pp. 1396-1400. 